\section{KOMPASS}

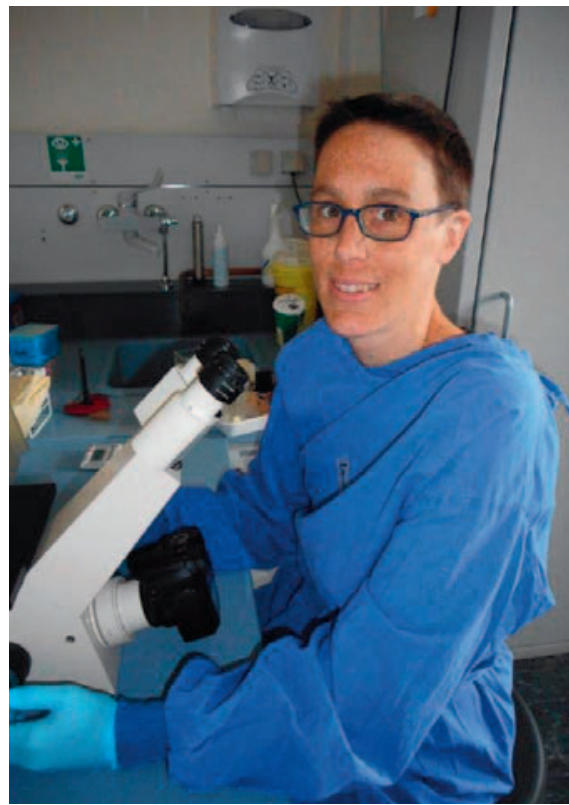

\title{
Dr. Laura Steenpaß
}

\section{Trägerin des Deutschen Förderprogramms für Augenheilkunde 2016}

\section{FORSCHUNGSSCHWERPUNKT}

- Entwicklung und Anwendung zellulärer Modelle zur Erforschung des genomischen Imprintings

\author{
AKADEMISCHER WERDEGANG \\ - Wissenschaftliche Mitarbeiterin und \\ Gruppenleiterin am Institut für Human- \\ genetik, Essen \\ - PostDoc im Labor von Dr. Denise Barlow, \\ Center of Molecular Medicine, Wien \\ - Promotion in Genetik, Universität Wien \\ - Studium der Biologie, Freiburg i.Br.
}

Welche Hypothese lag dem ausgezeichneten Forschungsprojekt «Differenzierung humaner embryonaler Stammzellen in neurale Retina: ein In-vitro-Modell für das Retinoblastom» zugrunde?

Unser Ausgangspunkt war weniger eine Hypothese, als das Vorhaben, ein In-vitro-Modell für das Retinoblastom zu entwickeln, da es bisher kein adäquates humanes Modell für diese Erkrankung gibt. Nach erfolgreicher Etablierung wollen wir das Modell dann für vielfältige, hypothesengetriebene Projekte einsetzen.

Was war das Hauptergebnis des Forschungsprojekts?

Wir haben erfolgreich humane embryonale Stammzellen erzeugt, die Mutationen im Retinoblastom-Gen (RB1) tragen, entweder auf einem oder auf beiden Allelen. Diese Zellen differenzieren wir momentan in neurale Retina.

Was hat Sie während der Arbeit oder nach Auswertung der Ergebnisse überrascht?

Überrascht hat mich, dass die Stammzellen mit der Veränderung im RB1-Gen keine beschleunigte Proliferation zeigen, d.h. Stammzellen reagieren auf den Verlust von $R B 1$ anders als retinale Vorläuferzellen. Dies zeigt, wie wichtig das richtige zelluläre Modell ist.

Worin liegt die Relevanz Ihrer Ergebnisse für die praktische, klinische Arbeit eines Ophthalmologen?

Unser Projekt ist momentan rein grundlagenwissenschaftlich orientiert. Wenn das Modell aber einmal etabliert ist, könnten wir damit Voraussagen über den molekularen Einfluss verschiedener Mutationen im RB1-Gen treffen. Dies könnte dann für die genetische Beratung und eventuell auch für Therapieoptionen relevant sein. Unser Stammzell-Modell kann natürlich auch für andere Fragestellungen, die die Wirkungsweise von RB1 betreffen, interessant sein. Besonders natürlich dadurch, dass sich mit Stammzellen frühe Entwicklungs- und Differenzierungsprozesse nachvollziehen lassen.

\section{Welche Fortschritte haben Sie seit der Auszeichnung erzielen können?}

Die Auszeichnung hat es uns überhaupt erst ermöglicht, dieses Projekt in Angriff zu nehmen. Ohne diese finanzielle Unterstützung wäre es uns nicht so schnell gelungen, die Stammzellen zu verändern und an der Etablierung der vergleichenden Differenzierung zu arbeiten.

Womit schaffen Sie für sich selbst und für Ihr Umfeld einen Ausgleich zu Ihrem Einsatz für die Forschung?

Als Ausgleich zur Laborarbeit versuche ich möglichst viel Zeit draußen zu verbringen, sei es beim Joggen oder Radfahren oder bei Unternehmungen mit meinen Kindern und meiner Familie.

\section{ANGABEN ZUM PREIS}

Das Deutsche Förderprogramm für Augenheilkunde ist eine Initiative der Bayer Vital GmbH. Ziel ist es, innovative wissenschaftliche Forschungsprojekte zu unterstützen, die der langfristigen Verbesserung der Behandlungsmöglichkeiten von Patienten mit Augenerkrankungen dienen. Jährlich wird eine Projektförderung bis max. 50000 EUR an innovative Konzepte und Projektideen in der Augenheilkunde mit Schwerpunkt auf retinalen Augenerkrankungen vergeben.

\section{KARGER}

Fax +497614520714 information@karger.com www.karger.com

\section{(c) 2017 S. Karger GmbH, Freiburg}

Dr. Laura Steenpaß

Institut für Humangenetik

Universitätsklinikum Essen

Hufelandstraße 55, 45127 Essen, Deutschland

Laura.Steenpass@uk-essen.de 